\title{
Better Radiographic Reduction and Lower Complication Rates With Combined Coracoclavicular and Acromioclavicular Ligament Reconstruction Than With Isolated Coracoclavicular Reconstruction
}

\author{
Jordan D. Walters, M.D., Anthony Ignozzi, B.S., Francis Bustos, M.D., \\ Brian C. Werner, M.D., and Stephen F. Brockmeier, M.D.
}

Purpose: To determine whether combined acromioclavicular (AC) ligament reconstruction and coracoclavicular (CC) ligament reconstruction without bone tunnels would improve radiographic reduction maintenance and complication rates for type III to V AC dislocations. Methods: This single-institution retrospective study analyzed all patients who underwent a hybrid synthetic/graft wrap CC reconstruction without tunnels with additional AC reconstruction/repair from January 2013 to August 2019. This 26-patient cohort was compared with a 1:1 sex-and age-matched control group who underwent CC reconstruction without AC reconstruction. CC distances on postoperative radiographs were compared with normal contralateral shoulders. Results: Of the 93 patients who underwent AC reconstructive surgery during this time period, 26 patients (96\% male) met the inclusion criteria. The AC/CC cohort had $23.5 \%$ type III injuries, $23.1 \%$ type IV injuries, and $53.8 \%$ type $\mathrm{V}$ injuries, similar to the control group. Final radiographs of the operative shoulder's CC distance were (mean \pm standard deviation) $0.9 \pm 4.0 \mathrm{~mm}$ greater than that of the contralateral shoulder $(9.6 \pm 8.7 \mathrm{~mm}) \mathrm{in}$ the $\mathrm{AC} /$ CC cohort. Final radiographs of the operative shoulder's coracoclavicular distance were $4.0 \pm 4.7 \mathrm{~mm}$ greater than that of the contralateral shoulder $(13.3 \pm 9.3 \mathrm{~mm})$ in the CC control group, a significant difference $(P=.014)$. The AC/CC reconstruction group had fewer patients with a loss of reduction $>5 \mathrm{~mm}(11.5 \%$ versus $38.5 \%, P=.025)$. The complication rate in the CC control group was higher than in the AC/CC cohort ( $30.7 \%$ versus $7.7 \%, P=.035)$. The reoperation rate was also greater in the CC control group ( 8 versus $1, P=.010)$. Conclusion: This cohort study shows that the addition of AC reconstruction to CC reconstruction using synthetic tapes/grafts or allograft tissues without bone tunnels significantly improves durable radiographic outcomes, diminishes complication rates, and improves reoperation rates. Level of Evidence: III, retrospective comparative study.

S cromioclavicular (AC) dislocation continues to confound the orthopedic community as a notoriously difficult injury to treat with or without surgery. The Rockwood classification remains central to communication and treatment determination regarding this injury. Type I, II, and often III injuries are managed

From the Department of Orthopaedic Surgery, University of Virginia Health System (J.D.W., F.B., B.C.W., S.F.B.) and the University of Virginia School of Medicine (A.I.), Charlottesville, Virginia, U.S.A.

The authors report the following potential conflicts of interest or sources of funding: B.C.W. reports personal fees, Arthrex; grants, Biomet, Flexion Therapeutics. S.F.B. reports personal fees, Arthrex; paid consultant, presenter, or speaker, research support, and personal fees, Biomet; IP royalties, paid consultant, presenter, or speaker, research support, and personal fees, Exactech; IP royalties and paid consultant, presenter, or speaker, International Society of Arthroscopy, Knee Surgery, and Orthopaedic Sports Medicine; board or committee member, MidAtlantic Shoulder and Elbow Society; board or committee member, publishing royalties, and financial or material support, Techniques in Shoulder and Elbow Surgery; editorial or governing board and conservatively, and type IV, V, and VI injuries (with some type III injuries) are mostly managed operatively. ${ }^{1}$ Controversy continues for all aspects of care, including operative versus nonoperative decisions, surgical timing and technique, rehabilitation and return to sport, need for concomitant surgery, etc.

personal fees, Zimmer. Full ICMJE author disclosure forms are available for this article online, as supplementary material.

Received April 1, 2020; accepted October 12, 2020.

Address correspondence to Jordan D. Walters, MD, Department of Orthopaedic Surgery, University of Virginia Health System, 515 Ray C Hunt Dr \#1100, Charlottesville,VA 22903,U.S.A. E-mail: jordan.walters1183@ gmail.com

(C) 2021 THE AUTHORS. Published by Elsevier Inc. on behalf of the Arthroscopy Association of North America. This is an open access article under the CC BY-NC-ND license (http://creativecommons.org/licenses/by-nc-nd/4.0/). 2666-061X/20417

https://doi.org/10.1016/j.asmr.2020.10.009 
Regarding type III, IV, and even V injuries, some studies have found no advantage of operative management in physical and mental health 2 years after injury. ${ }^{2-5}$ However, other studies have shown significant health improvement at 2-year follow-up with surgery versus conservative management. ${ }^{6}$ Although nonoperative treatment avoids the potential complications inherent to surgery, persistent pain, functional limitation, and scapular dyskinesis with its associated symptoms occurs in $>70 \%$ of patients treated conservatively. $^{7}$

Historically, AC joint injuries have been treated with $>150$ surgical variations, including Weaver-Dunn reconstruction, suture fixation, screw fixation, hook plate fixation, tendon allograft/autograft reconstruction, synthetic graft fixation, etc., focused on reconstruction of the coracoclavicular (CC) ligaments. ${ }^{8-11}$ However, recurrent dynamic anterior/posterior translation occurs in nearly half of patients after isolated CC reconstruction. ${ }^{12,13}$ Combined AC/CC ligament reconstruction has been advocated to improve shoulder girdle kinematics and maintain stability better than isolated CC reconstruction. ${ }^{14,15}$

One institution previously reported on its experience with surgical management of acute and chronic AC dislocations using isolated CC reconstruction techniques, citing a notable $30 \%$ overall complication rate and a $22 \%$ loss of reduction rate, roughly $20 \%$ of those complications being fractures in either the clavicle or coracoid. ${ }^{16}$ Since that time, combined AC/CC ligament reconstruction open techniques that avoid both coracoid and clavicle tunnels have become our preferred surgical approach. We sought to determine whether combined AC ligament reconstruction and CC ligament reconstruction without bone tunnels would improve radiographic reduction maintenance and complication rates for type III to V AC dislocations. We hypothesized that the combined AC/CC reconstruction would improve radiographic reduction and decrease postoperative complications.

\section{Methods}

This study (institutional review board approval HSR \# 21984) involved a retrospective review at a single institution of all operations for an acute or chronic closed AC dislocation using CC ligament reconstruction with or without AC ligament reconstruction from January 2013 to August 2019 using Current Procedural Terminology codes 23550 (open treatment of acromioclavicular dislocation, acute or chronic) or 23552 (open treatment of acromioclavicular dislocation, acute or chronic; with fascial graft [includes obtaining graft]). Inclusion criteria were skeletal maturity, preoperative and postoperative radiographic evidence of injury and reconstruction with inclusion of the contralateral shoulder for comparison, and specific documentation of reconstruction of both the $\mathrm{CC}$ and $\mathrm{AC}$ ligaments in the operative report. Patients undergoing concomitant procedures or revision AC/CC reconstruction were not excluded as long as the reconstruction met appropriate technique criteria. Surgeries for both acute $(<6$ weeks from injury) and chronic ( $\geq 6$ weeks from injury) AC dislocations were included. An age- and sex-matched control group was established in a 1:1 ratio from patients who underwent isolated CC reconstruction without additional AC reconstruction at our institution. Each patient was matched within 3 years of the corresponding study patient's age.

The CC distance measurement based on a standing anteroposterior (AP) Zanca shoulder/bilateral AC joint radiograph was used to determine postoperative displacement/loss of reduction compared with the contralateral normal distance (Fig 1). Measurements were made from the superior coracoid in a vertical line to the inferior clavicle using our institution's digital picture archiving and communication system according to standard validated technique by an orthopedic surgery sports medicine fellow not involved in the care of these patients. ${ }^{17}$ Complications and reoperations were determined based on chart review.

Several reconstructive methods were used during this study. For the study cohort, 2 surgeons (B.C.W. and S.F.B.) performed a hybrid synthetic graft plus autograft/allograft tendon open CC reconstruction procedure using either a synthetic graft/screw construct (Lockdown ${ }^{\mathrm{TM}}$, Worcestershire, UK) or a novel Fibertape ${ }^{\mathrm{TM}}$ (Arthrex, Naples, FL) construct with dual tapes using a luggage tag configuration wrapped around the coracoid and stabilized with a DogBone ${ }^{\mathrm{TM}}$ (Arthrex) superior to the clavicle (Fig 2). No coracoid drill holes were used. Semitendinosus or gracilis allograft (LifeNet Health, Virginia Beach, VA), wrapped around the coracoid and tied down over the clavicle, was used for 21 of 26 patients $(81 \%)$ as additional fixation. The remaining 5 patients had semitendinosus autograft harvested from the ipsilateral leg for the procedure in similar fashion. No drill holes were used in the clavicle for graft placement; graft tissue was wrapped around the coracoid and the clavicle and secured with suture fixation. The remaining limbs of graft tissue were then pulled over and secured to the superior AC ligaments, which were previously dissected free, or secured to the superior acromion with a suture anchor (2.9-mm Pushlock biocomposite anchor; Arthrex) for AC ligament reconstruction (Fig 3). Distal clavicle excision was performed per surgeon's discretion based on the reducibility of the joint. Layered closure was performed with a secure deltotrapezial fascial closure. Postoperatively, a sling was worn for 6 weeks, and then gradual rehabilitation was performed with a goal of return to activities by 4 to 6 months. 
Fig 1. (A) Preoperative upright bilateral standing anteroposterior (AP) acromioclavicular (AC) joint radiograph showing a left type $\mathrm{V}$ AC dislocation. (B) Postoperative upright bilateral standing AP AC joint radiograph showing appropriate reduction of left AC joint after combined hybrid coracoclavicular (CC) reconstruction using a Lockdown device with AC reconstruction with both left and right CC distances of $8.3 \mathrm{~mm}$.

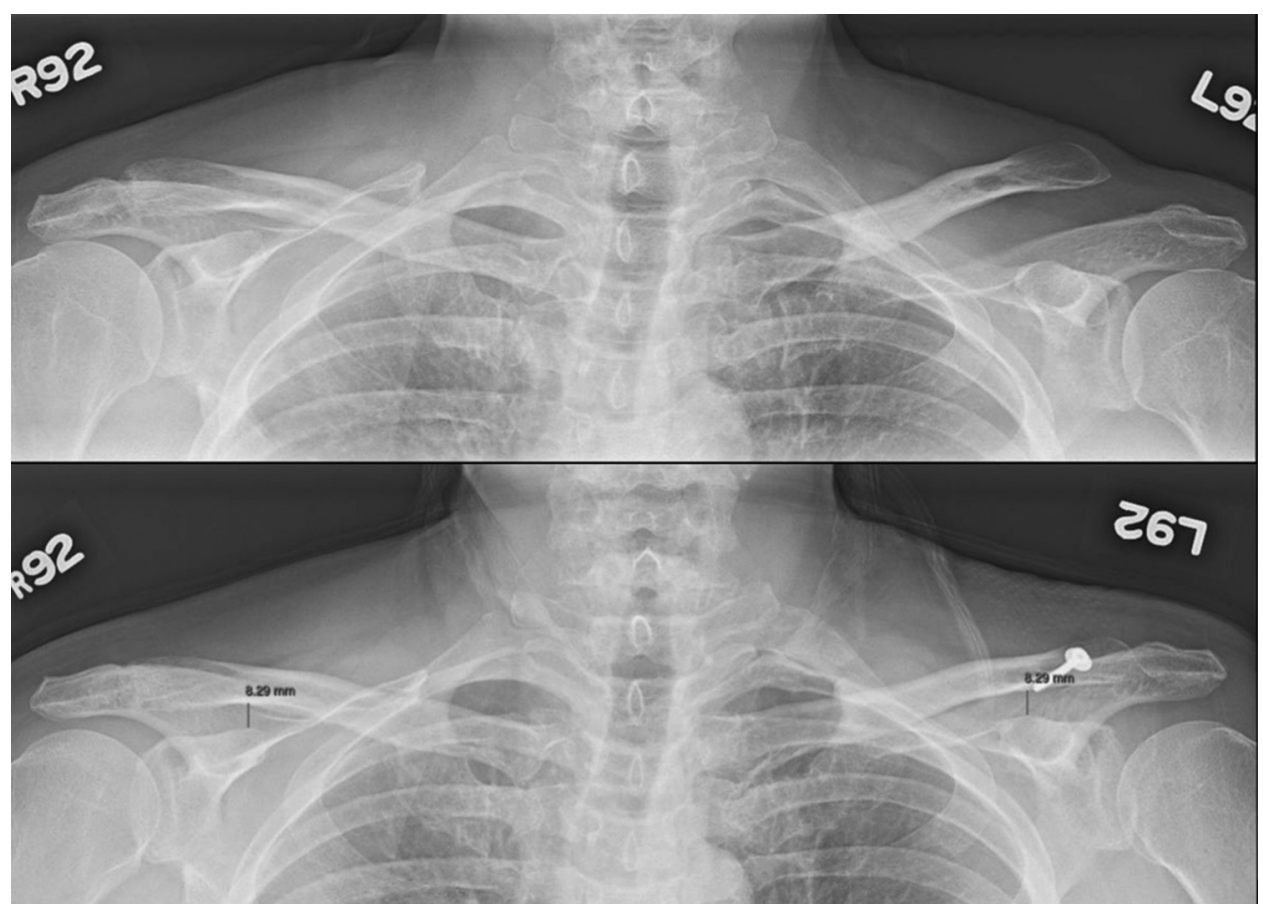

The control group consisted of multiple methods of CC reconstruction, including isolated synthetic graft (Lockdown), semitendinosus allograft/autograft reconstruction with drill tunnels through the clavicle, suspension button reconstruction, and allograft CC reconstruction augmented with hook plate fixation, that were performed during the same time period. Six surgeons (including B.C.W. and S.F.B.) at our institution performed the operations in this control group. No AC-specific reconstruction was performed for any patient in the control group. Layered closure and postoperative rehabilitation were performed similarly to the study group's methods. Routine hook plate hardware removal was not considered a reoperation.

Statistical analysis was performed using SPSS software (release 2017; IBM SPSS Statistics for Windows, version 25.0. IBM Corp., Armonk, NY). Chi-squared analysis or Fisher exact testing was performed for all comparisons as required for the data. $P$ values $<.05$ were considered significant. A post hoc analysis showed $61.9 \%$ power.

\section{Results}

A total of 93 patients underwent open treatment for an acromioclavicular dislocation during the study period from January 2013 through August 2019. Sixtyseven patients underwent isolated CC reconstruction without the addition of $\mathrm{AC}$ reconstruction and were excluded. Twenty-six patients met all inclusion criteria and were included in our AC/CC reconstruction cohort. Age and sex matching were performed in a 1:1 fashion to create an isolated CC reconstruction control group consisting of 26 patients who underwent surgery during the same time period. Each group's mean follow-up time was 6 months (range 6 weeks to 2.5 years).

Regarding demographics, the mean ages of each patient group were nearly identical (AC/CC cohort mean \pm standard deviation, $36.5 \pm 15.8$ years versus CC control, $36.5 \pm 16.3$ year, $P=1.000$ ). The vast majority of patients in each group were male $(96.2 \%$ in AC/CC and $92.3 \%$ in CC control, $P=.552$ ). The mean body mass index (BMI) of the AC/CC cohort was lower than that of the CC control group $\left(24.6 \pm 2.9 \mathrm{~kg} / \mathrm{m}^{2}\right.$ versus $\left.27.2 \pm 4.5 \mathrm{~kg} / \mathrm{m}^{2}, P=.017\right)$. Operations involving the dominant arm were similar between groups $(53.8 \%$ in AC/CC cohort versus $42.3 \%$ in CC control group, $P=$ $.405)$. Active tobacco smokers made up $11.5 \%$ of the AC/CC cohort and $34.6 \%$ of the CC control group, but the difference was not significant $(P=.071)$ (Table 1$)$.

Describing injuries, there were no significant differences regarding the specific Rockwood classification types between groups. There were 6 type III injuries $(23.1 \%), 6$ type IV injuries $(23.1 \%)$, and 14 type V injuries $(53.8 \%)$ in the AC/CC cohort. There were 4 type III injuries (15.4\%), 4 type IV injuries (15.4\%), and 18 type $\mathrm{V}$ injuries $(69.2 \%)$ in the control group. No significant differences were seen in the proportion of acute versus chronic injuries in each group. There were 10 acute injuries $(38.5 \%)$ treated surgically in the AC/ CC cohort, and there were 13 acute injuries $(50.0 \%)$ treated surgically in the CC control group $(P=.402)$. Similar rates of concomitant arthroscopic procedures 


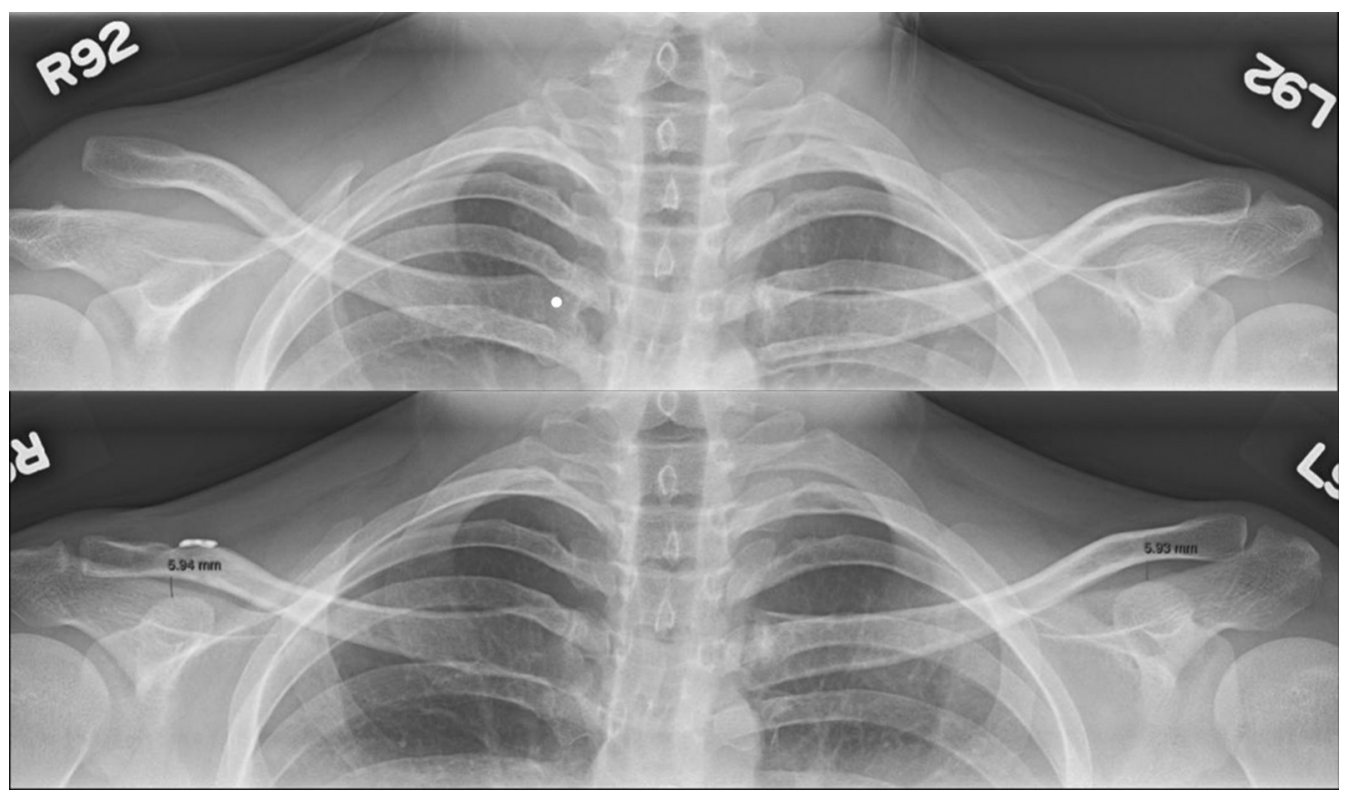

Fig 2. (A) Preoperative upright bilateral standing anteroposterior (AP) acromioclavicular (AC) joint radiograph showing a right type V AC dislocation. (B) Postoperative upright bilateral standing AP AC joint radiograph showing appropriate reduction of right AC joint after combined hybrid coracoclavicular (CC) reconstruction using double Fibertape plus Dogbone fixation with AC reconstruction with both left and right CC distances of $5.9 \mathrm{~mm}$.

including rotator cuff repair, biceps tenodesis, and labral repair were performed in each group (Table 1).

Considering outcomes, final radiographs of the operative shoulder's CC distance were $0.9 \pm 4.0 \mathrm{~mm}$ greater than the $\mathrm{CC}$ distance of the contralateral shoulder (9.6 versus $8.7 \mathrm{~mm}$ ) in the AC/CC cohort. Final radiographs of the operative shoulder's CC distance were $4.0 \pm 4.7 \mathrm{~mm}$ greater than the CC distance of the contralateral shoulder ( 13.3 versus $9.3 \mathrm{~mm}$ ) in the CC control group, significantly greater than the difference in the AC/CC cohort $(P=.014)$. Three patients $(11.5 \%)$ in the study cohort had a loss of reduction $>5 \mathrm{~mm}$, whereas 10 patients $(38.5 \%)$ in the control group had such a loss in reduction $(P=.025)$. There were fewer complications in the AC/CC cohort than in the CC control group ( 2 versus $8, P=.035$ ). The complications in the AC/CC cohort were a clavicle fracture in a ski accident 7 weeks postoperatively and 1 coracoid partial osteolysis that occurred from a prominent Lockdown screw in the clavicle. CC control group complications included acute loss of fixation in 2 patients, significant distal clavicle osteolysis leading to loss of fixation, postoperative wound infection, postoperative adhesive capsulitis of the shoulder in 2 patients, clavicle fracture, and a coracoid fracture. The reoperation rate was also greater in the $\mathrm{CC}$ control group than in the AC/CC cohort ( 8 versus $1, P=.010$ ) (Table 2). The only reoperation in the AC/CC cohort was a Lockdown screw removal; notably, this patient had maintenance of reduction before and after hardware removal. Reoperations in the CC control group included a CC ligament reconstruction with hook plate augmentation, clavicle open reduction/internal fixation (ORIF) with autograft and subsequent bone grafting revision, wound debridement and irrigation, shoulder arthroscopic lysis of adhesions and manipulation in 2 patients, clavicle ORIF, and coracoid ORIF. Routine planned hardware removals for hook plates $(n=6)$ were not considered reoperations related to complications and thus were not included in this analysis.

\section{Discussion}

The most important findings of this study were that combined AC and CC reconstruction better maintained radiographic reduction, with significantly decreased

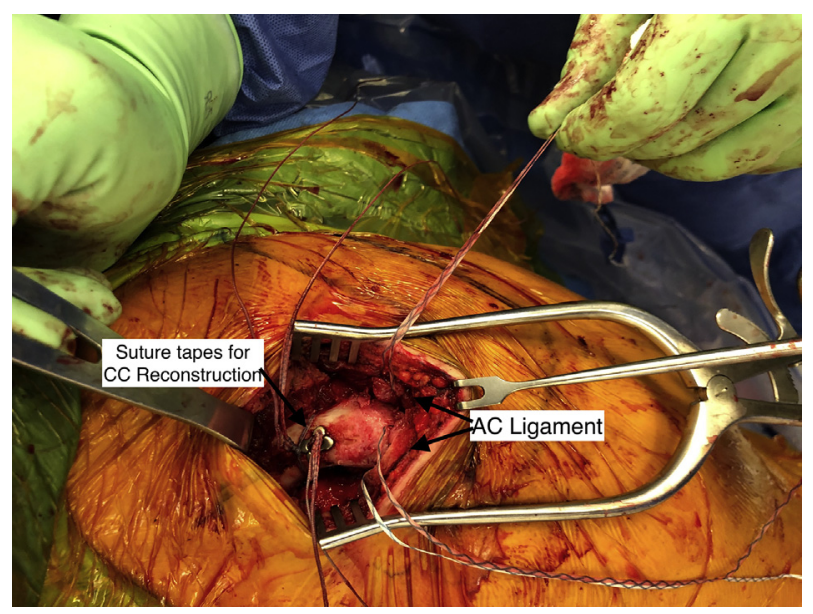

Fig 3. Dissection of acromioclavicular (AC) ligaments in preparation for AC reconstruction with suture anchor incorporating graft limbs. 
Table 1. Study cohort and matched control demographics and characteristics

\begin{tabular}{lccr}
\hline \multicolumn{1}{c}{ Variable } & CC/AC Study Cohort $(\mathrm{n}=26)$ & CC Control Cohort $(\mathrm{n}=26)$ & Statistical Comparison $(P$ Value $)$ \\
\hline Demographics & & & \\
Age $(\mathrm{y})$ & $36.5 \pm 15.8$ & $36.5 \pm 16.3$ & .000 \\
BMI $\left(\mathrm{kg} / \mathrm{m}^{2}\right)$ & $24.6 \pm 2.9$ & $27.2 \pm 4.5$ & .017 \\
Male sex & $25(96.2)$ & $24(92.3)$ & .552 \\
Dominant arm & $14(53.8)$ & $11(42.3)$ & .405 \\
Nonsmoker & $21(80.8)$ & $15(57.7)$ & .071 \\
Rockwood classification & & & .482 \\
Type III & $6(23.1)$ & $4(15.4)$ & .482 \\
Type IV & $6(23.1)$ & $18(69.2)$ & .254 \\
Type V & $14(53.8)$ & $13(50.0)$ & .402 \\
Chronicity & $10(38.5)$ & $13(50.0)$ & .402 \\
Acute & $16(61.5)$ & $3(11.5)$ & .000 \\
Chronic & & $5(19.2)$ & .083 \\
Concomitant procedures & $3(11.5)$ & $1(3.8)$ & \\
Rotator cuff repair & $4(15.4)$ & $5(19.2)$ &
\end{tabular}

Data are mean \pm SD or $\mathrm{n}(\%)$.

AC, acromioclavicular; BMI, body mass index; CC, coracoclavicular.

rates of complications and reoperations compared with a CC reconstruction control group that did not include $\mathrm{AC}$ reconstruction for the treatment of type III to $\mathrm{V}$ AC dislocations. These findings suggest that combined AC/ CC reconstruction may provide improved stability for this difficult injury.

Historically, many surgeons have debated the merits of nonoperative and operative treatment using various techniques for type III to V AC dislocations. A metaanalysis comparing operative to nonoperative treatment showed minimal functional differences, although cosmesis and radiographic reduction favored surgery whereas earlier return to work and lower complication rates favored nonoperative management. ${ }^{18} \mathrm{~A}$ randomized clinical trial between nonoperative management and hook plate application for AC dislocation types III to V showed better radiographic reduction in the operative group but worse early clinical scores and more complications. ${ }^{19}$ However, specific rehabilitation is recommended to overcome the scapular dyskinesis that can result from AC dislocations. Although it typically resolves within 6 to 12 weeks, AC dislocationassociated scapular dyskinesis can take up to 12 months to resolve. ${ }^{20}$ One systematic review has shown a return to sport rate of $94 \%$ to $100 \%$ after operative treatment, which is an improvement from the $83 \%$ to $94 \%$ rate of return to sport seen with conservative management. ${ }^{21}$

When surgery is indicated based on shared physical/ patient decision making, it is important to limit negative outcomes. Because $77 \%$ of patients with type V injuries treated nonoperatively can return to work and activities, it is the surgeon's duty to avoid complications that could worsen the final result. ${ }^{22}$ One study of isolated CC reconstruction cited a $94.5 \%$ return to work rate but noted an $18.5 \%$ revision rate and a $34.6 \%$ rate of persistent symptoms. ${ }^{23}$ Postoperative complication rates $>20 \%$ and loss of reduction rates $>30 \%$ have

Table 2. Radiographic outcomes and complications

\begin{tabular}{lccc}
\hline \multicolumn{1}{c}{ Variable } & CC/AC Study Cohort $(\mathrm{n}=26)$ & CC Control Cohort $(\mathrm{n}=26)$ & Statistical Comparison $(P$ Value $)$ \\
\hline $\begin{array}{l}\text { Radiographic } \\
\text { Final CC distance difference from } \\
\quad \text { contralateral }(\mathrm{mm})\end{array}$ & $0.9 \pm 4.0$ & $4.0 \pm 4.7$ & .014 \\
$\begin{array}{l}\text { Complications } \\
\text { Overall }\end{array}$ & $2(7.7)$ & & .035 \\
Reoperation & $1(3.8)$ & $8(30.8)$ & .010 \\
Revision CC ligament & $0(0.0)$ & $2(7.7)$ & .149 \\
Hardware removal & $1(3.8)$ & $5(19.2)$ & .083 \\
Infection/incision and drainage & $0(0.0)$ & $1(3.8)$ & .313 \\
Adhesive capsulitis & $0(0.0)$ & $2(7.7)$ & .149 \\
Fracture & $1(3.8)$ & .552 & $.7 .7)$ \\
\hline
\end{tabular}

Data are mean \pm SD or $\mathrm{n}(\%)$.

AC, acromioclavicular; CC, coracoclavicular. 
been found in multiple studies. ${ }^{24,25}$ One meta-analysis showed an overall loss of reduction rate of $20.8 \%$ and complication rate of $14.2 \%$ with a revision rate of $9.5 \% .{ }^{11}$ Surgical complications include infection, loss of reduction, suture breakage with button migration, graft stretch, clavicular osteolysis, hardware pullout, adhesive capsulitis, and neuropathy. ${ }^{26,27}$ Fractures of the clavicle or coracoid account for $5 \%$ to $20 \%$ of these complications, so we avoid tunnels altogether, opting for a graft wrapped around the clavicle and coracoid similar to Millett et al. ${ }^{28-30}$ The clavicle fracture in the study group was suffered by a patient who engaged in sport activities early against medical advice. We believe that tunnel avoidance in the AC/CC cohort was key to limiting the major fracture complications seen in our control group that did include such drilling techniques.

Our understanding continues to improve of the spectrum of AC dislocation pathology. The Acromioclavicular Joint Instability Score (ACJI) is a recent classification that uses Alexander views of the shoulder to capture dynamic horizontal instability in addition to the vertical instability noted on standard bilateral standing AP AC joint views. ${ }^{31}$ A Rockwood type III injury subclassification considers a type IIIA to be stable and IIIB to be unstable based on the presence or absence of an overriding distal clavicle with cross-arm adduction AP shoulder radiographs. ${ }^{32}$ The posterior translation test, which evaluates the amount of posterior shift of the distal clavicle compared with the contralateral AC joint, also suggests horizontal instability. ${ }^{33}$ Because the AC ligaments are key to horizontal stability, these studies suggest that AC ligament reconstruction in addition to CC ligament reconstruction may improve stability and outcomes.

Although 1 biomechanical study failed to show improved fixation with additional AC joint reconstruction, most studies have shown greater horizontal and vertical stability plus decreased stress on the CC fixation. ${ }^{14,32,34,35}$ Using arthroscopically assisted continuous loop fixation, combined AC/CC fixation has shown decreased rates of dynamic posterior translation compared with isolated CC fixation. ${ }^{12}$ A semitendinosus autograft weave technique to reconstruct the CC and AC ligaments has shown promising outcomes as well. ${ }^{33}$ Similar to our study, Tauber et al. ${ }^{36}$ found that AC/CC reconstruction with a triple-bundle technique had a lower complication rate than the isolated CC single-bundle reconstruction $(16.7 \%$ versus $35.7 \%)$. Beitzel et al. ${ }^{14}$ showed that suturing 1 excess limb of the CC reconstruction allograft anteriorly, posteriorly, and superiorly around the AC joint best limited translation in the horizontal and vertical planes when comparing AC reconstruction techniques.

Related to postoperative outcomes, glenohumeral joint pathology has been found in $15 \%$ to $53 \%$ of patients who suffer type III to V AC dislocations. ${ }^{37-40}$ We therefore obtain magnetic resonance imaging (MRI) preoperatively of our AC dislocation patients and perform diagnostic arthroscopy before open AC/CC reconstruction if any doubt exists for rotator cuff injury, biceps/SLAP injury, etc., to address all pathology of the shoulder girdle. There were no significant differences in intra-articular pathology or arthroscopic concomitant procedures between groups in this study.

Operative timing and its relationship to graft use is another area of debate for AC dislocations. Although tendon graft CC reconstruction is generally accepted for chronic AC dislocation treatment, acute treatment with a graft may or may not be beneficial with regard to loss of reduction. One group showed that graft reconstructions had a $42 \%$ displacement rate, whereas continuous loop button constructs without a graft had a $23 \%$ displacement rate. ${ }^{10}$ Although some biomechanical studies suggest that such loop button CC reconstruction techniques decrease translation of the AC joint compared with coracoid graft sling techniques, the testing frames are unable to recreate all of the muscle forces and fascial healing associated with clinical healing and rehabilitation environments. ${ }^{41}$ Another group showed a $47 \%$ loss of reduction rate with a $20 \%$ complication rate for acute AC dislocations treated with autograft tendon $\mathrm{CC}$ reconstruction using a single tunnel, believed to be due to elongation of the graft tissue. ${ }^{28}$ Based on these studies, our hybrid techniques set the CC fixation length with inelastic suture tape or synthetic graft and use graft tissue as backup fixation to incorporate collagen for ultimate tissue healing, limiting elongation concerns.

Multiple techniques were used for our control group, including CC reconstruction with graft. However, a systematic review has shown no complication rate or failure rate differences between ligament/tendon transfers such as Weaver-Dunn, autograft/allograft CC reconstruction, and synthetic CC reconstruction grafts. ${ }^{8}$ Hook plate fixation was used as backup fixation to CC reconstructions using allograft tissue for some in our control group. Such extra fixation would be expected to improve overall reduction maintenance in this group, thus strengthening the study findings. No complications were noted related to hook plates. Routine hook plate removal was not included as part of our reoperation rate for the control group.

\section{Limitations}

Limitations do exist for this study. This retrospective review has bias risks inherent to such work, including limited power. Our operative cohort included patients from 2 surgeons, but patients from 6 surgeons were included in the control group. Although this diversity may limit comparability, we believe that this increases the generalizability of our findings. We created as homogeneous a study group as possible, including only 
those patients who specifically underwent a strong repair or reconstruction of the AC ligament tissues in addition to stable hybrid CC reconstruction without bone tunnels. Our follow-up time period is variable, but this represents orthopaedic practice in the real-world setting. Some of these limitations exist because surgical treatment of AC dislocations is relatively uncommon, as evidenced by our small study groups in a group of subspecialty sports medicine surgeons in a highvolume tertiary practice setting. We included both acute and chronic AC dislocations in this study, but there were no significant overall treatment timing differences between the matched groups. Despite the statistically significant difference in BMI between groups, we do not believe that a difference of 2.6 within a healthy BMI range is clinically meaningful. Also, a wide range of age was evident for the patients in this study. However, all patients were symptomatic from their AC dislocation and desired to return to activity and/or work.

\section{Conclusion}

This study shows that the addition of AC reconstruction to CC reconstruction using synthetic tapes/ grafts and/or allograft tissues without bone tunnels significantly improves durable radiographic outcomes, diminishes complication rates, and improves reoperation rates.

\section{References}

1. Rockwood CA. Injuries to the acromioclavicular joint. In: Rockwood CA, Green DP, eds. Fractures in Adults. 2nd ed., 1 Philadelphia: JB Lippincott Co, 1984;860.

2. Tamaoki MJS, Lenza M, Matsunaga FT, Belloti JC, Matsumoto MH, Faloppa F. Surgical versus conservative interventions for treating acromioclavicular dislocation of the shoulder in adults. Cochrane Database Syst Rev 2019;10: CD007429.

3. Chang N, Furey A, Kurdin A. Operative versus nonoperative management of acute high-grade acromioclavicular dislocations-A systematic review and meta-analysis. J Orthop Trauma 2018;32:1-9.

4. Mah J, The Canadian Orthopaedic Trauma Society. General health status after nonoperative versus operative treatment for acute, complete acromioclavicular joint dislocation-Results of a multicenter randomized clinical trial. J Orthop Trauma 2017;31:485-490.

5. Longo UG, Ciuffreda M, Rizzello G, et al. Surgical versus conservative management of type III acromioclavicular dislocation-A systematic review. Br Med Bull 2017;122: $31-49$.

6. Natera Cisneros L, Sarasquete Reiriz J, Abat F, et al. Acute unstable acromioclavicular joint injuries: Quality of life comparison between patients managed operatively with a coracoclavicular suspension device arthroscopically placed versus patients managed non-operatively. Eur Orthop Traumatol 2015;6:343-355.
7. Gumina S, Carbone S, Postacchini F. Scapular dyskinesis and SICK scapula syndrome in patients with chronic type III acromioclavicular dislocation. Arthroscopy 2009;25: 40-45.

8. Borbas P, Churchill J, Ek ET. Surgical management of chronic high-grade acromioclavicular joint dislocations: A systematic review. J Shoulder Elbow Surg 2019;28: 20311-22038.

9. Weaver JK, Dunn HK. Treatment of acromioclavicular injuries, especially complete acromioclavicular separation. J Bone Joint Surg Am 1972;54:1187-1194.

10. Lee BK, Jamgochian GC, Syed UAM, et al. Reconstruction of acute acromioclavicular (AC) joint dislocations with or without tendon graft-A retrospective comparative study. Arch Bone Joint Surg 2019;7:239-245.

11. Gowd AK, Liu JN, Cabarcas BC, et al. Current concepts in the operative management of acromioclavicular dislocations-A systematic review and meta-analysis of operative techniques. Am J Sports Med 2019;47: 2745-2758.

12. Maziak N, Audige L, Hann C, Minkus M, Scheibel M. Factors predicting the outcome after arthroscopically assisted stabilization of acute high-grade acromioclavicular joint dislocations. Am J Sports Med 2019;47: $2670-2677$.

13. Hann C, Kraus N, Minkus M, Maziak N, Scheibel M. Combined arthroscopically assisted coraco- and acromioclavicular stabilization of acute high-grade acromioclavicular joint separations. Knee Surg Sports Traumatol Arthrosc 2018;26:212-220.

14. Beitzel K, Obopilwe E, Apostolakos J, et al. Rotational and translational stability of different methods for direct acromioclavicular ligament repair in anatomic acromioclavicular joint reconstruction. Am J Sports Med 2014;42: 2141-2148.

15. Saier T, Venjakob AJ, Minzlaff P, Fohr P, Lindell F, Imhoff $A B$, Vogt S, Braun S. Value of additional acromioclavicular cerclage for horizontal stability in complete acromioclavicular separation: A biomechanical study. Knee Surg Sports Traumatol Arthrosc 2015;23:1498-1505.

16. Milewski MD, Tompkins M, Giugale JM, et al. Complications related to anatomic reconstruction of the coracoclavicular ligaments. Am J Sports Med 2012;40: 1628-1634.

17. Zumestein MA, Schiessl P, Ambuehl B, et al. New quantitative radiographic parameters for vertical and horizontal instability in acromioclavicular joint dislocations. Knee Surg Sports Traumatol Arthrosc 2018;26:125-135.

18. Smith TO, Chester R, Pearse EO, Hing CB. Operative versus non-operative management following Rockwood grade III acromioclavicular separation: A metaanalysis of the current evidence base. J Orthop Traumatol 2011;12: 19-27.

19. The Canadian Orthopaedic Trauma Society. Multicenter randomized clinical trial of nonoperative versus operative treatment of acute acromio-clavicular joint dislocation. J Orthop Trauma 2015;29:479-487.

20. Carbone S, Postacchini F, Gumina S. Scapular dyskinesis and SICK syndrome in patients with a chronic type III acromioclavicular dislocation. Results of rehabilitation. Knee Surg Sports Traumatol Arthrosc 2015;21:1473-1480. 
21. Kay J, Memon M, Alolabi B. Return to sport and clinical outcomes after surgical management of acromioclavicular joint dislocation: A systematic review. Arthroscopy 2018;34:2910-2924.

22. Dunphy TR, Damodar D, Heckmann ND, Omid R, Hatch GF. Functional outcomes of type V acromioclavicular injuries with nonsurgical treatment. J Am Acad Orthop Surg 2016;0:1-7.

23. Porschke F, Schnetzke M, Studier-Fischer S, Gruetzner PA, Guehring T. Return to work after acromioclavicular joint stabilization-A retrospective case control study. J Orthop Surg Res 2019;14:1-7.

24. Choi NH, Lim SM, Lee SY, Lim TK. Loss of reduction and complications of coracoclavicular ligament reconstruction with autogenous tendon graft in acute acromioclavicular dislocations. J Shoulder Elbow Surg 2017;26:692-698.

25. Shin SJ, Kim NK. Complications after arthroscopic coracoclavicular reconstruction using a single adjustableloop-length suspensory fixation device in acute acromioclavicular joint dislocation. Arthroscopy 2015;31:816-824.

26. Cook JB, Krul KP. Challenges in treating acromioclavicular separations: Current concepts. J Am Acad Orthop Surg 2018;26:669-677.

27. Struhl S, Wolfson TS. Continuous loop double endobutton reconstruction for acromioclavicular joint dislocation. Am J Sports Med 2015;43:2437-2444.

28. Martetschlager F, Horan MP, Warth RJ, Millett PJ. Complications after anatomic fixation and reconstruction of the coracoclavicular ligaments. Am J Sports Med 2013;41: 2896-2903.

29. Cook JB, Tokish JM. Surgical management of acromioclavicular dislocations. Clin Sports Med 2014;33:721-737.

30. Millett PJ, Horan MP, Warth RJ. Two-year outcomes after primary anatomic coracoclavicular ligament reconstruction. Arthroscopy 2015;31:1962-1973.

31. Kraus N, Hann C, Gerhardt C, Scheibel M. Dynamic instability of the acromioclavicular joint: A new classification for acute AC joint separation. Obere Extremitat 2018;13:279-285.

32. Virk MS, Apostolakos J, Cote MP, et al. Operative and nonoperative treatment of acromioclavicular dislocation: A critical analysis review. J Bone Joint Surg Rev 2015;3: e5-el7.
33. Garofalo R, Ceccarelli E, Castagna A, et al. Open capsular and ligament reconstruction with semitendinosus hamstring autograft successfully controls superior and posterior translation for type $\mathrm{V}$ acromioclavicular joint dislocation. Knee Surg Sports Traumatol Arthrosc 2017;25: 1989-1994.

34. Weiser L, Nuchtern JV, Sellenschloh K, et al. Acromioclavicular joint dislocations: Coracoclavicular reconstruction with and without additional direct acromioclavicular repair. Knee Surg Sports Traumatol Arthrosc 2017;25: 2025-2031.

35. Sumanont S, Nopamassiri S, Boonrod A, et al. Acromioclavicular joint dislocation: A Dog Bone button fixation alone versus Dog Bone button fixation augmented with acromioclavicular repair-A finite element analysis study. Eur J Orthop Surg Traumatol 2018;28:1095-1101.

36. Tauber M, Valler D, Lichtenberg S, Magosch P, Moroder P, Habermeyer. Arthroscopic stabilisation of chronic acromioclavicular joint dislocations. Triple versus singlebundle construction. Am J Sports Med 2016;44:482-489.

37. Pauly S, Gerhardt C, Haas NP, Scheibel M. Prevalence and pattern of glenohumeral injuries among acute high-grade acromioclavicular joint instabilities. Knee Surg Sports Traumatol Arthrosc 2009;17:513-517.

38. Markel J, Scwartin T, Malcherczyk D, Peterlein CD, Ruccholtz S, El-Zayat BF. Concomitant glenohumeral pathologies in high-grade acromioclavicular separation (type III - V). BMC Musculoskeletal Disorders 2017;18: 439-444.

39. Jensen G, Millett PJ, Tahal DS, Al Ibadi M, Lill H, Katthagen JC. Concomitant glenohumeral pathologies associated with acute and chronic grade III and grade V acromioclavicular joint injuries. Int Ortho (SICOT) 2017;41: 1633-1640.

40. Arrigoni P, Brady PC, Zottarelli, et al. Associated lesions requiring additional surgical treatment in grade 3 acromioclavicular joint dislocations. Arthroscopy 2014;30: 6-10.

41. Grantham C, Heckmann N, Wang L, Tibone JE, Struhl S, Lee TQ. A biomechanical assessment of a novel double Endobutton technique versus a coracoid cerclage sling for acromioclavicular and coracoclavicular injuries. Knee Surg Sports Traumatol Arthrosc 2016;24:1918-1924. 